\title{
TDAH - TRANSTORNO DE DÉFICIT DE ATENÇÃO E HIPERATIVIDADE E A MAXIMIZAÇÃO PSICOPATOLOGICA DE SEUS SINTOMAS
}

Evelyn de Paula Souza, Rosiane de Fatima Ponce.

Universidade Estadual - UNESP, Programa de Pós-Graduação em Educação, Presidente Prudente, SP. E-mail: eve.paula@hotmail.com.br. Agência de fomento: CAPES

\section{RESUMO}

Visto o crescente aumento de diagnósticos de Transtorno de Déficit de Atenção e sua hegemônica concepção em sociedade. Com tal preocupação centramos nosso trabalho no objetivo de compreender e verificar, no cenário acadêmico, as quantas andam as pesquisas que compreendem o TDAH a partir de uma perspectiva biológica e de Teoria Histórico-cultural. A metodologia constou na busca bibliográfica de teses e dissertações com a temática TDAH, nos programas de pós-graduação de Universidades do Estado de São Paulo e Bancos de Periódicos de 2005 a 2015. Os resultados obtidos de 153 trabalhos acadêmicos demonstram que conceitos de individualização de individuo, biologicista. Ou seja, majoritariamente os trabalhos apontam que o TDAH é um 'problema' do indivíduo, seja por questões de natureza biológica/genética ou por situações do entorno da 'família' da criança. Tais pesquisas, no geral, disseminam uma concepção orgânica de homem. Concluímos, que apenas uma visão Histórico-social de Homem e uma visão crítica sobre cenário educacional pode nos encaminhar para o desenvolvimento de processos de transformação e minimização de problemas escolares.

Palavras-chave: TDAH, Teoria Histórico-cultural, Pedagogia Histórico-Critica, Dificuldade de Aprendizagem.

\section{ADHD - DISORDER DEFICIT AND ATTENTION AND HYPERACTIVITY MAXIMIZATION PSYCHOPATHOLOGICAL SYMPTOMS OF ITS .}

\begin{abstract}
Given the increasing Deficit Disorder diagnoses of Attention and its hegemonic design in society. With this concern we focus our work in order to understand and verify, in the academic scenario, many go the polls which include ADHD from a biological perspective and Theory Historical-cultural. The methodology consisted in a bibliographic search of theses and dissertations with thematic ADHD, programs graduate of the State University of São Paulo and 2005 Journal Banks to 2015. The results of 153 academic studies have shown that individualization concepts individual, biologicist. Le mainly the studies indicate that ADHD is a 'problem' of the individual, whether for reasons of biological / genetic or surrounding situations of 'family' of criança.Tais research in general, spread a man's organic design. We conclude that only a history-social vision of man and a critical view of educational setting can lead us to the development of manufacturing processes and minimizing school problems.
\end{abstract}

Keywords: ADHD Theory Historico -Cultural, Education Historico - Critica, Difficulty learning.

\section{INTRODUÇÃO}

Destacamos que o objetivo deste artigo está em apresentar alguns dos dados de uma pesquisa de Mestrado, em andamento, realizado no Programa de Pós-graduação em Educação (PPGE/FCT/UNESP) sobre a temática 'TDAH' e processos de medicalização da infância em Presidente Prudente. Inicialmente, a pesquisa visou verificar, no cenário acadêmico, como as pesquisas acadêmicas nos últimos anos (de 2005-2015) abordam o tema 'TDAH' e quais são as 'vertentes teóricas' que predominam nestes trabalhos de pesquisas.

Visando iniciar com a definição do que é o TDAH, destacamos que, a partir dos estudos já empreendidos na pesquisa de Mestrado, este fenômeno social, nos últimos anos, está se configurando hegemonicamente dentro de uma 
concepção biologicista. Assim, optamos por apresentar o conceito de acordo com o que encontramos em 'manuais' de orientação aos pais, responsáveis, professores e sociedade de forma em geral. Ao longo deste artigo, pretendemos refletir sobre as algumas das explicações de fracasso escolar embutidas no processo crescente de medicalização e psicopatologização da infância.

Segundo o Manual Diagnóstico e Estatístico de Transtornos Mentais (DSMV-IV), instrumento mundial de orientação para profissionais da área de saúde mental, considerado a maior referência para análise de transtornos mentais, traz o TDAH dentre a categoria de Transtornos Invasivos do Desenvolvimento sem Outra Especificação. De acordo com o manual, tal categoria é utilizada para distinguir transtornos que prejudicam de forma invasiva e severa o desenvolvimento e a interação social da criança, onde as principais características que cerceiam o diagnóstico de TDAH se encontram na perseverança dos sintomas de desatenção, impulsividade e hiperatividade. Explicitando que, a desatenção pode ser identificada durante momentos escolares, sociais, familiares e até mesmo profissionais, o que faz com que o individuo cometa erros, tenha dificuldade em organizar atividade, seja imprudente, ocorrendo muitas vezes confusão e fracasso em suas atividades escolares. Já o aspecto de hiperatividade manifesta-se principalmente pela inquietude de seus atos, visto nas crianças quando realizam corridas por lugares inapropriados, balanços frenéticos das pernas e mãos, falas em excesso, nível elevado de movimentos na carteira, entre outros. Por fim, a impulsividade identifica-se na impaciência de seus atos como respostas precipitadas, pegar objetos que não são permitidos, fazer palhaçadas, fazer falas inoportunas, em alguns casos levando até acidentes.

De acordo com a ABDA - Associação Brasileira de Déficit de Atenção, fundação criada em 1999 sem fins lucrativos para auxilio de pacientes, o 'TDAH' é um transtorno de causas genéticas e de ordem neurobiológica, se revelando em sintomas durante a infância e persistindo durante a fase adulta. As pesquisas de suas causas centram seus estudos na afirmação de que o transtorno é efeito de alterações na região frontal do cérebro com suas demais conexões, região esta, responsável pela inibição e controle do comportamento. Ainda segundo a associação, estudos apontam que o dano frontal está exatamente no sistema de substâncias químicas que controlam as sinapses dessa região, mas especificamente nos seus neurotransmissores. Lembrando que seus apontamentos se baseiam nos mais renomados estudos e consensos de médicos e psicólogos.

Ainda explicitando as compreensões biológicas do fenômeno 'TDAH', Cirio (2008) em busca de proposta para pais e professores, relata que o 'TDAH' é um problema de saúde mental e que por tal disfunção neurológica apresenta danos no controle de seus impulsos e na atividade motora. E que causas: "[...] podem estar relacionadas a aspectos orgânicos, emocionais, sociais e genéticos, tornando-se um caso de difícil diagnóstico" (p.13).

Em outra citação da autora sobre a verificação desse diagnóstico, esclarece que:

Existem escalas que
descrevem os sintomas, mas
estas não podem servir
como instrumentos isolados
para a realização do
diagnóstico, que deve ser
baseado nas atitudes da
criança em casa, na escola,
no consultório, nas
informações dos pais e
professores e no trabalho de
equipe com outros
profissionais. (CIRIO, 2008,
p.13).

Ou seja, é realmente difícil analisar corretamente o diagnóstico de 'TDAH' segundo as especificidades que temos discutido até aqui, visto que a análise e características de seu transtorno se baseiam paulatinamente em sintomas comportamentais, os quais são levados em consideração em ambientes sociais, principalmente comportamentos dentro do âmbito escolar, servindo de justificativas para o não aprender. Entretanto, o nível de dificuldade do laudo desse diagnóstico não foi empecilho algum para o aumento do número de crianças diagnosticadas, o que no mínimo pressupõe algum tipo de desconfiança quanto à veracidade desses transtornos.

O nosso objetivo está em compreender o 'TDAH' como sendo um fenômeno social e não como um "transtorno" que pertence a um único indivíduo, como se o mesmo estivesse doente ou 
Ihe faltando alguma função cognitiva. No geral, o 'TDAH' está sendo configurado como sendo um transtorno mental por diversos órgãos de saúde e que vem aumentando a cada dia o número de laudos realizados sobre as crianças que apresentam comportamentos "inadequados" e, principalmente, com dificuldades de aprendizagens escolares. $\mathrm{O}$ que tentamos alertar, na pesquisa de Mestrado em andamento, é a maximização de problemáticas médicas para o âmbito de aspectos educacionais e sociais. $\mathrm{Na}$ situação de naturalização do 'não-aprender' os conteúdos escolares como sendo situações atreladas ao fator 'biológico' das crianças e de suas famílias. Tentamos alertar e refletir sobre as interelações humanas, no âmbito social e político, as quais devem ser levadas em consideração quando nos propomos a compreensão de processos de formação humana.

\section{METODOLOGIA}

A fim de verificar as presentes discussões históricas dos conceitos do fenômeno 'TDAH', assim como, a sua defesa no cenário acadêmico universitário, realizamos buscas bibliográficas em bancos de teses e dissertações que tivessem como eixo principal a temática 'TDAH'. Destes trabalhos, tínhamos como objetivo analisar as opções teórico-metodológica-filosóficas para levantar a existência de trabalhos que compreendam o TDAH como um fenômeno social e a partir de uma concepção histórico-social de homem/sociedade, que é a opção teórica da pesquisa de mestrado que realizamos, tendo como aporte teórico principal a Teoria Histórico-cultural e a Pedagogia Histórico-crítica.

Em uma primeira etapa limitamos nossas análises ao índice de universidades públicas do Estado de São Paulo que continham os programas de pós-graduação em Educação e Psicologia. A busca por teses e dissertações foi realizada em um intervalo de publicação de 10 anos (de 2005 a 2015). As universidades Públicas selecionadas foram UNESP'S, USP, UFSCAR E UNICAMP, com exceção de uma Universidade Privada PUC (Campinas e São Paulo). Ressaltamos que nem todas as instituições selecionadas possuem em sua instituição o desenvolvimento de ambas as pós-graduações de Educação e Psicologia, o que levou a analisar em alguns casos apenas um programa na instituição de ensino.

Ao longo do levantamento e análise destas pesquisas, compreendemos que seria importante levantar pesquisas também no banco de dados da Capes e no banco de periódico Parthenon (de 2005 a 2015), na temática do TDAH utilizando os unitermos: Medicalização, TDAH, Transtorno de Aprendizagem e Dificuldade de Aprendizagem. Esclarecemos que obedecendo a parâmetros de 'filtros de buscas' dos próprios bancos de dados selecionamos apenas em teses e dissertações de língua portuguesa. Esta segunda etapa foi elaborada com o principal intuito de minimizar erros durante a primeira etapa nos bancos de dissertações e teses das Universidades Públicas do Estado de São Paulo e reforçar nossas concepções quanto ao cenário de estudo do tema discutido na pesquisa de Mestrado, em andamento.

\section{RESULTADOS}

$\mathrm{Na}$ análise de buscas da primeira etapa, encontramos ao todo 51 pesquisas, entre teses e dissertações, sendo delas 19 pesquisas em programas de pós-graduação em Educação dentre as 5 universidades analisadas USP, UNICAMP, UNESP, PUC e UFSCAR, resultando em 33 pesquisas no programa de Pós-graduação em Psicologia também dentre as universidades citadas.

Como resultado prelimiar, destacamos que encontramos que a temática TDAH compareceu em trabalhos que visavam discutir as seguintes temáticas: 
Tabela 1. Mapeamento de Temáticas.

\begin{tabular}{|c|c|c|c|c|c|}
\hline Temática & $\begin{array}{l}\text { Área da } \\
\text { Educação }\end{array}$ & $\begin{array}{c}\text { Área da } \\
\text { Psicologia }\end{array}$ & Total & Teses & Dissertações \\
\hline Fracasso Escolar & 8 & 2 & 10 & 4 & 6 \\
\hline $\begin{array}{l}\text { Dificuldade de } \\
\text { Aprendizagem }\end{array}$ & 6 & 9 & 15 & 7 & 8 \\
\hline $\begin{array}{l}\text { Concepção de } \\
\text { TDAH }\end{array}$ & 3 & 12 & 15 & 3 & 12 \\
\hline Medicalização & 1 & 2 & 3 & - & 3 \\
\hline $\begin{array}{l}\text { Demais } \\
\text { temáticas } \\
\text { (discalculia, } \\
\text { exclusão,etc) }\end{array}$ & 1 & 8 & 9 & 1 & 8 \\
\hline
\end{tabular}

Percebemos que, no geral, a grande maioria dos trabalhos que englobam a discussão do fenômeno 'TDAH' está mais presente nos Programas de pós-graduação em Psicologia, apesar de a temática discutida estar voltada ao "Fracasso Escolar" e "Dificuldade de Aprendizagem", conforme dados acima na tabela. Destes, apenas dois analisaram o 'TDAH' a partir da Teoria histórico-cultural, identificando o sujeito diagnosticado como produto sócio histórico e defendendo a concepção de TDAH enquanto um fenômeno social.

Ainda dos resultados dessa primeira etapa, encontramos dos 51 trabalhos acadêmicos, que apenas 19 que se atentava a discutir o "diagnóstico" TDAH como foco central de seus trabalhos. Destas dezenove produções, um total de 11 se encaixaram na perspectiva psicopatológica do fenômeno, cabe esclarecer que as definimos nesta categoria devido à defesa de que o Transtorno de Déficit de Atenção é uma "doença" que se encontra no próprio indivíduo, mais precisamente devido a disfunções neurobiológicas, o que coloca na própria criança a culpa pelo não aprendizado.

Nos resultados obtidos na segunda etapa de buscas de teses e dissertações envolvendo a discussão temática do 'TDAH', utilizando os unitermos chaves no banco de dados da Capes e Parthenon, encontramos: 32 pesquisas com a palavra-chave "TDAH", 32 pesquisas com a palavra-chave "Transtorno de Aprendizagem", 27 pesquisas com a palavra-chave "Dificuldade de Aprendizagem" e 11 com "Medicalização". Lembrando que tais resultados alcançam uma maior amplitude de população, avançando para além de Universidades do Estado de São Paulo, porém englobando apenas aquelas cedidas ao portal desses bancos de dados. Entretanto, apesar do aumento de numero em relação à etapa um, identificamos apenas 1 trabalho que parte da teoria histórico-cultural e que relaciona as dificuldades comportamentais e de aprendizagem da criança a questões históricas, políticas e sociais.

Assim como fizemos na primeira etapa, realizamos, também, a análise sobre as fundamentações teórico-metodológicas do tema 'TDAH' presentes nestas pesquisas encontradas. Nesse sentido, dos 102 trabalhos encontrados nos bancos de dados da CAPES e PARTHENON, um total de 52 tinham como objetivo de discussão a temática central do TDAH. Destes 52 identificamos 48 teses e dissertações que defendem uma concepção de TDAH a partir da psicanalise, psicologia comportamental e psicopatológica, atribuindo o Transtorno ao caráter puramente biológico.

\section{DISCUSSÃO}

No geral, das análises efetuadas na pesquisa de Mestrado, em andamento, as publicações de teses e dissertações não estão muito além do que o reflexo do cenário educacional, que tem mostrado um aumento do uso de medicalização no campo escolar e de velhas justificativas médicas, que apontam que o TDAH está no indivíduo como justificativa ao "não aprender", contudo, e o que mais nos inquieta, é o crescente número de crianças que não conseguem aprender.

Saviani (2012) nos alerta que a centralidade de problemas que são, no geral, do âmbito educacional no próprio indivíduo, isso ocorre no Brasil desde a Escola Nova. Para o autor, nesta época buscaram-se avanços educacionais para fazer frente à Escola Tradicional, apontando o sujeito 'marginalizado', 
os quais eram àqueles que não estavam dentro do sistema educacional e àqueles que mesmo dentro do sistema educacional mantinham-se em condição de analfabetos, como individuo rejeitado da sociedade. Ou seja, se na Escola Tradicional a centralidade do sistema educacional estava no professor, no seu saber, na Escola Nova essa demanda voltou-se para os alunos, que eram considerados sujeitos principais do processo de ensino-aprendizagem:

Forja-se, então, uma pedagogia que advoga um tratamento diferencial a partir da "descoberta" das diferenças individuais. Eis a "grande descoberta" os homens são essencialmente diferentes; não se repetem; cada indivíduo é único. Portanto, a marginalidade não pode ser explicada pelas diferenças entre os homens, quaisquer que sejam: não apenas diferenças de cor, de raça, de credo ou de classe, o que já era defendido pela pedagogia tradicional; mais também diferenças no domínio de conhecimento na participação do saber, no desempenho cognitivo. Marginalizados são os "anormais", isto é, os desajustados e inadaptados de todos os matizes. (SAVIANI, 2012, p.8).

Ironicamente, o que temos, já naquela época, é o inicio da justificativa de que se as crianças não aprendem, é porque a 'culpa' é delas. Ou seja, está no próprio indivíduo o fracasso escolar, pois se lhe é garantida a inserção na escola e este não aprende os conhecimentos fornecidos na instituição, a culpa só pode estar na criança. Entram em cena, então, os conceitos de 'anormais', de 'visão maturacional' e 'construtivista' de homem, que defendem que são as crianças que não conseguem se adaptar ao meio educacional.

Duarte (2001), discutindo a temática do "aprender a aprender" nos ideários educacionais, afirma que tanto no cenário construtivista quanto da escola nova, essa temática apresenta quatro ideários valorativos que intensificam a hegemonia de visões individualistas e maturacionais de individuo. O primeiro se encontra na concepção na valorização das aprendizagens individuais, aquelas que, realiza por si mesmo, sem auxilio ou transmissão por parte do professor. A segunda conceitua a importância de o aluno desenvolver um próprio meio de se apropriar dos conhecimentos, de construí-lo. $\mathrm{O}$ terceiro se coloca no posicionamento de que a atividade educativa centra-se na impulsão das necessidades e interesses dos próprios alunos. Por fim, a quarta que acredita que a educação deva promover atividades educativas que preparem o individuo para a sociedade, para acompanharem suas mudanças. Há a todo o momento uma defesa de educação que não somente atribui ao aluno toda a carga de aprendizagem e desenvolvimento como também desqualifica a funcionalidade do professor como educador. Estranhamente "superamos" a escola nova e suas perspectivas dentro da escola, entretanto porque tais aspectos apresentados acima não nos parecem estranhos no âmbito escolar? Com convicção, afirmamos que se deve ao fato de que tais conceitos ainda permanecem engendrados no contexto educacional, justificando e culpabilizando indivíduos por seus fracassos escolares. Pois, como nos afirma Meira:

Diante da pergunta "por que os alunos continuam na escola, mas não aprendem?" a Psicologia apresenta inúmeros argumentos pretensamente científicos, todos eles focados nos indivíduos: disfunções orgânicas; traços de personalidade; capacidade intelectual; habilidades e competências

perceptivo-motoras;

problemas emocionais; comportamentos

inadequados; carências culturais; dificuldades de linguagem; desnutrição; despreparo para enfrentar as tarefas da escola; falta de apoio das famílias; "desagregação" familiar; conflitos uma unidade de natureza ideológica: a ênfase dada á adaptação dos 
indivíduos á escola e á sociedade. (MEIRA, 2011 p.95).

Ênfase esta que não foge em nenhum momento das discussões já realizadas. Ou seja, a partir de justificativas médicas, psicológicas e tratamentos farmacológicos colocamos um fim, uma solução, na discussão sobre as crianças que não aprendem ou que apresentam comportamentos fora do "padrão" adequado. 0 problema se encontra no âmbito educacional e o primeiro passo que tomamos é buscar compreende-lo através de um viés psicopatologizante e biologicista do sujeito em processo de aprendizagens. Destacamos não desconsideramos a importância dos estudos psicológicos e médicos sobre o desenvolvimento humano, o que discutimos é a hegemonia de compreensões que transformam questões sociais, políticas e culturais em processos biologizantes. É alto o número de crianças diagnosticadas e a hegemônica concepção psicopatológica do TDAH: "Em síntese, a escola é para todos, mas nem todos podem aproveitar essa oportunidade em decorrência de problemas individuais." (MEIRA, 2011p. 94).

\section{CONCLUSÕES}

Compreendendo o desenvolvimento humano a partir da Teoria Histórico-Cultural, identificamos o sujeito não somente como produto de suas construções individuais, nem tão pouco resultado de uma sequência maturacional de desenvolvimento, tida como padrão de normalidade. Para Meira (2011), a dimensão biológica para a Teoria Histórico-Cultural não é descartada quando se estuda a formação do individuo, muito pelo contrário, é a primeira condição para que o homem seja "candidato" ao processo de humanização. Porém, sua formação só irá se constituir a partir das possibilidades de vivenciar processos de interelação com meio social, simbólico e humanizado, acarretando em apropriações e objetivações humanas/materiais. Ou seja, é através da aprendizagem que o homem se desenvolve e poderá ser tornar um 'ser humano humanizado', pois suas formações são históricas e não apenas de cunho genético.

Compreendemos, assim, que precisamos compreender o fenômeno TDAH dentro de uma compreensão histórica e cultural para que possamos de fato engendrar transformações nos processos de ensino dos conteúdos escolares e, assim, enfrentarmos a situação de medicalização da infância sem que seja considerada com necessária e natural aos sujeitos com dificuldades de aprendizagens escolares.

\section{REFERÊNCIAS}

ABDA. ASSOCIAÇÃO BRASILEIRA DE DÉFICIT DE ATENÇÃO E HIPERATIVIDADE. Banco de dados. Disponivel em: <http://www.tdah.org.br/>. Acesso: Jul 2016.

BARKLEY, R. A. Transtorno de Déficit de Atenção/Hiperatividade (TDAH): guia completo para pais, professores e profissionais da saúde. Porto Alegre: Artmed, 2002.

CIRIO, R.R. Transtorno de Déficit de Atenção/Hiperatividade: Propostas para Pais e Professores. São Paulo: Vetor, 2008.

DUARTE, N. Vygotski e o "Aprender a Aprender": Criticas ás apropriações Neoliberais e Pós-Modernas da Teoria Vygotskiana. Campinas, SP: Autores Associados, 2001.

MEIRA, M.E.M. Incluir para continuar excluindo: a produção da exclusão na educação brasileira á luz da psicologia Histórico-cultural. In: FACCI,M.G.D; MEIRA, M.E.M; TULESKI, S.C. A Exclusão dos "Incluídos": Uma crítica da Psicologia da Educação á Patologização e Medicalização dos Processos Educativos. Maringá: Eduem, 2011. p.91-132.

MEIRA, M.E.M. A medicalização e a produção da exclusão na educação brasileira à luz da Psicologia Histórico-Cultural. In: ENCONTRO NACIONAL DA ABRAPSO. 15., Maceió. Disponível em:

$<$ http://www.abrapso.org.br/siteprincipal/images Annais XVENABRAPSO/3.\%20a\%20medicaliza\%C 7\%C30\%20e\%20a\%20produ\%C7\%C30\%20da\%20 exclus\%C30\%20na\%20educa\%C7\%C30\%20brasile ira\%20\%C0\%20luz\%20da\%20psicologia\%20hist\% D3rico-cultural.pdf>. Acesso em: jul. 2016.

SAVIANI, D. Escola e Democracia. Campinas: Autores Associados, 2012.

Recebido para publicação em 23/08/2016

Revisado em 02/09/2016

Aceito em 29/09/2016 